\title{
Direct puncture versus run up cervical myelography with iopamidol: a comparison of side effects, EEG changes and radiographic quality
}

\author{
P MACPHERSON, E TEASDALE, AP McGEORGE \\ From the Departments of Neuroradiology and Neurology, Institute of Neurological Sciences, Southern \\ General Hospital, Glasgow, UK
}

SUMMARY A series of cervical myelograms performed by direct puncture resulted in almost identical incidence of side effects, more contrast within the skull, more frequent EEG abnormalities and only slightly better radiographic quality than in a comparable series of patients in whom the contrast was run up from the lumbar region.

It is generally believed that cervical myelographic by run up from the lumber region is more likely to result in intracranial spillage of contrast than when the examination is done by direct puncture..$^{1-4}$ The frequency of side effects such as headache and nausea and the occurrence of seizures are considered to be proportional to the amount of contrast entering the skull. ${ }^{14-7}$ Accordingly, it has been the practise in this institute to give prophylactic anticonvulsants when the contrast was run up but not if introduced by direct cervical puncture. As part of our evaluation of iopamidol we studied two groups of patients, 20 having direct puncture and 20 having run up cervical myelography. EEG was performed before and after the myelogram and any changes noted, the relative clinical side effects were assessed, the quantitiy of contrast within the skull was estimated radiographically and by $\mathrm{CT}$ and the radiographic quality of the myelograms compared.

\section{Patients}

40 patients in the series gave informed consent for additional EEG and CT scan examinations. Patients with a history of seizures or of receiving potentiating drugs would have been excluded, but none presented during the trial period. When the cervical region was the site of clinical interest the investigation was done by direct puncture at the $\mathrm{C} 1-2$ level and where there was doubt about the site of

Address for reprint requests: Dr $P$ Macpherson, Institute of Neurological Sciences, Southern General Hospital, 1345 Govan Rd, Glasgow, G51 4TF, UK.

Received 18 December 1982 and in revised form 15 April 1983. Accepted 7 May 1983 the lesion, run up myelography was performed with the patient in the prone postion. By chance, clinical presentation of 40 consecutive patients resulted in the allocation of 20 to each group: 10 male and 10 female patients in the run up, and eight male and 12 female patients in the direct puncture group. The ages are comparable in the two groups. Following upon our previous practise, run up patients were given weight-related dosage of sodium valproate; a loading dose (1500 $\mathrm{mg}$ for patient weight $40-$ $55 \mathrm{~kg} ; 2000 \mathrm{mg}$ for $56-70 \mathrm{~kg} ; 2500 \mathrm{mg}$ for $71-85 \mathrm{~kg}$ ) at 8 pm on the evening before the examination and maintenance doses $(700 ; 1000 ; 1200 \mathrm{mg}$ respectively) at $8 \mathrm{am}$ and $8 \mathrm{pm}$ on the day of examination.

\section{Method}

On the day prior to examination an EEG was performed. All the myelograms were performed by either PM or ET and great care was taken to prevent the extension of contrast intracranially, though when clinically indicated (10 direct puncture and 10 run up patients) a supine view was obtained with contrast in the cisterna magna. All punctures were performed with a 20 gauge needle and $10 \mathrm{ml}$ of CSF withdrawn for diagnostic purposes. The run up patients received $10 \mathrm{ml}$ of iopamidol in a concentration of 300 $\mathrm{mgI} / \mathrm{ml}$ and direct puncture patients $7 \mathrm{ml}$ of $250 \mathrm{mgI} / \mathrm{ml}$. At the end of the examination the radiologist completed a questionnaire noting details of the precedure and any immediate side effects experienced by the patient. A radiographer unaware into which category the patient had been allocated interviewed the patient and nursing staff at approximately 24 and 48 hours after the myelogram noting any complications. The patients were instructed not to lie flat until after the post-myelogram EEG.

Six to seven hours after the injection of contrast each patient had a repeat EEG while sitting, followed by a CT scan in the supine position. On the latter, the density of iopamidol in the subarachnoid spaces was measured at 
three levels: ambient cisterns, Sylvian fissures, and cortical sulci.

From the meylogram, views of the cervical spine and skull base were randomised by one radiologist author and reviewed "blind" by the other, The quality of the contrast in the cervical region was scored as "satisfactory", "good" or "excellent": the volume which had entered the interpeduncluar cistern was graded as "nil", "slight", "moderate"or "marked".

\section{Results}

\section{SIDE EFFECTS}

The results are given in Table $1 \mathrm{a}$, and with the exception of nausea are identical in each group.

RADIOGRAPHIC CONTRAST WITHIN THE SKULL A lateral film of the skull taken at the end of the myelographic examination was available in 35 of the 40 patients. More contrast was present within the interpeduncular cisterns in the examinations performed by direct puncture-p $\sim 0.10$ (Table 1b).

\section{CONTRAST DENSITY WITHIN THE SKULL IN \\ HOUNSFIELD UNITS (HU)}

The contrast densities in the different compartments within the skull $6 \frac{1}{2}-71 / 2$ hours after the examination are shown in Table 1c. More contrast was present on average in the ambient cisterns, Sylvian fissures and cortical sulci in the direct puncture than in the run up group but the difference was significant only in the cortical sulci $(p \sim 0.05)$.

\section{EEG RESULTS}

Post-myelographic EEG tracings showed focal sharp waves in two of the direct puncture group and in one of the run up group; generalised sharp wave discharges were present in four of the direct puncture and two of the run up patients. In three of the

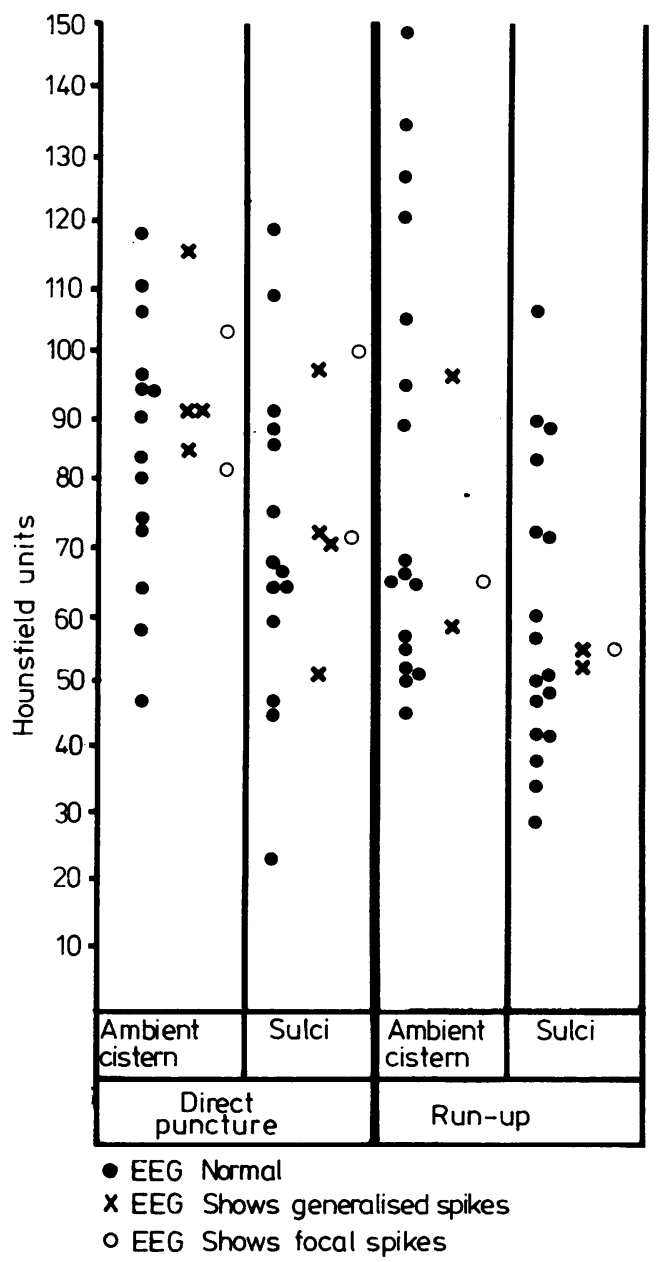

Fig The EEG results in relation to the contrast density (HU) within the ambient cisterns and cortical sulci.

Table 1 Comparison of side effects and of immediate and late contrast density within the skull in the direct puncture ( $N=$ 20) and run up $(N=20)$ groups.

(a) The incidence of side effects.

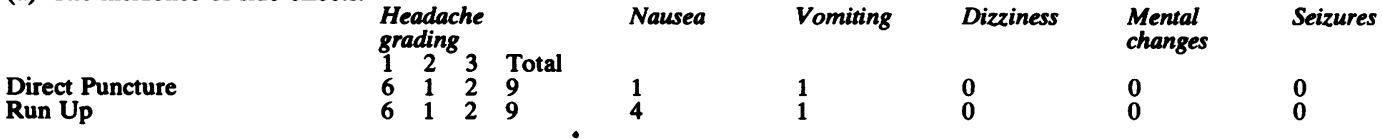

(b) Radiographic density of contrast within the interpeduncular cistern at the end of the myelogram.

\begin{tabular}{|c|c|c|c|c|c|}
\hline $\begin{array}{l}\text { Direct Puncture } \\
\text { Run Up }\end{array}$ & $\begin{array}{l}\text { No Filim } \\
1 \\
4\end{array}$ & $\begin{array}{c}\text { None } \\
6 \\
12\end{array}$ & $\begin{array}{l}\text { Slight } \\
3 \\
1\end{array}$ & $\begin{array}{l}\text { Moderate } \\
3 \\
1\end{array}$ & $\begin{array}{l}\text { Marked } \\
7 \\
2\end{array}$ \\
\hline
\end{tabular}

(c) The contrast density (HU) within the skull $6 \frac{1}{2}-7 \frac{1}{2}$ hours after the myelogram.

Direct Puncture Ambient cisterns Run Up 
patients with generalised discharges the premyelographic tracing had shown focal sharp waves. Two were in the direct puncture group; one suffered from chronic bronchitis and emphysema, had intermittent claudication and was hypothyroid, while the other had a family history of epilepsy. The third patient was in the run up group and at the time of the examination was clinically thyrotoxic because of thyroxin overdosage.

\section{RELATIONSHIP OF EEG CHANGES TO THE}

CONTRAST DENSITY IN THE AMBIENT CISTERNS

AND CORTICAL SULCI

In the direct puncture group no changes were seen in the five patients where the density of contrast in the ambient cisterns was below $80 \mathrm{HU}$. Of the 15 patients with higher density, six had sharp waves (two focal and four generalised). Of the nine patients where the density of contrast in the cortical sulci was under $70 \mathrm{HU}$ only one patient developed generalised sharp waves while of the 11 with higher density, five had sharp waves (two focal and three generalised). However, the above findings are not statistically significant.

In the run up group there was no relationship in the three patients with sharp waves (one focal and two generalised) with the density of contrast in either the ambient cistern or the cortical sulci.

\section{QUALITY OF RADIOGRAPHIC EXAMINATION IN THE CERVICAL REGION}

In the direct puncture group two were graded as "poor", 11 as "good" and seven as "excellent": while the corresponding figures for the run up group were four; 12 and four.

\section{Discussion}

\section{SIDE EFFECTS}

In a survey of 120 patients undergoing direct puncture cervical myelography with metrizamide, we found that the incidence of headache was $60 \%$, nausea $33 \%$, vomiting $26 \%$, dizziness $32 \%$; three patients developed mental changes and one generalised seizures. ${ }^{8}$ The corresponding figures in the direct puncture group in the present series using iopamidol were, with the exception of headache (45\%), all significantly less: nausea $5 \%(p<0.01)$ and vomiting $5 \%$, dizziness $0 \%(p<0.005)$. In the present run up group, the incidence and grading of headache, vomiting and dizziness was identical to that in the direct puncture group. Mental changes and seizures did not occur in this small series. In an interhospital survey using iopamidol, Bassi et al ${ }^{9}$ found the complication rates of run up myelography to average $41.5 \%$.
CONTRAST WITHIN THE SKULL

Despite the belief mentioned in the introduction, we found that significantly more contrast was detectable radiographically and by $C T$ in the direct puncture than in the run up group. Surprisingly, there was no direct correlation between the radiographic grading of intracranial contrast and the subsequent $\mathrm{HU}$ measurement.

\section{EEG}

Changes reported after metrizamide myelography have been classified into two groups: (a) short slow waves which are seen maximally 24 hours after the injection of contrast and which are not considered to have any clinical significance, (b) sharp waves which are detectable some hours after the examination and are considered to be a direct irritative effect on the cerebral cortex. ${ }^{1011}$ It is believed that the danger of seizures can be minimised by preventing metrizamide from reaching the cortex..$^{16}$

Lundervold and Sortland 6 found that the frequency of all types of EEG changes rose from 4-5\% where the upper limit of contrast as seen on a lateral film was in the cervical region or pontine cistern to $10 \%$ where it had reached the suprasellar cistern and to $50 \%$ where it was visible over the frontal cortex. Epileptiform spikes were however seen in only $4 \%$ of their patients. Some authors have reported that EEG changes do not occur after radiculography ${ }^{1213}$ but Standnes et al $^{4}$ reported that epileptiform sharp waves were seen in $10 \%$ of their radiculogram patients.

In our series using iopamidol, sharp waves were seen in six of $20(33 \%)$ of the direct puncture group and in three of the $20(16 \%)$ of the run up group. While not of statistical significance because of the small numbers it seems that direct puncture cervical myelography is as likely to result in epileptiform EEG activity as is run up myelography. It has been found that medication with diazepam before and after myelography was of no value in preventing EEG abnormalities ${ }^{6}$ and apparently it is not known if sodium valproate can protect against development of such sharp waves. Harrington et al $^{14}$ have shown that EEG changes do not occur as a result of lumbar puncture alone, lending proof to the belief that after myelography such abnormalities are a reaction to the contrast medium. However, we found no clear correlation between the CT attenuation and sharp wave activity, suggesting that individual sensitivity is an important factor.

The focal sharp wave activity occurring in three of our patients, is consistent with cortical irritation. In six patients generalised discharges suggest a centricephalic site of action although secondary subcortical activity is not ruled out. It may, therefore, be 
important to prevent contrast reaching the upper brain stem.

This study indicates that while a direct puncture cervical myelogram is likely to give marginally better quality radiographs it is more likely to result in contrast entering the subarachnoid spaces around the brain and to a greater chance of causing irritative EEG effects than a carefully performed examination carried out by running the contrast from the lumbar region. If there is a case for prophylactic anticonvulsants in the run up investigations then there must be a stronger case for their use in direct puncture myelography.

We are grateful to Dr B Whiting and Dr I Bone for their advice on the use of prophylactic anticonvulsant therapy and to Dr JP Ballantyne for advice on the EEG aspects of the study.

\section{References}

${ }^{1}$ Amundsen P. Metrizamide in cervical myelography. Acta Radiol [Diagn] (Stockh) 1977; Suppl 355:85-97.

${ }^{2}$ Cronqvist S, Brismar J. Cervical myelography with metrizamide. Acta Radiol [Diagn] (Stockh) 1977; Suppl 355:110-20.

${ }^{3}$ Sortland O, Skalpe IO. Cervical myelography by lateral cervical and lumber injection of metrizamide. Acta Radiol [Diagn] (Stockh) 1977; Suppl 355:154-63.

${ }^{4}$ Standnes B, Oftedal S-I, Weber H. Effect of levomepromazine on EEG and clinical side effects after lum- bar myelography with metrizamide. Acta Radiol [Diagn] (Stockh) 1982; 23:111-4.

${ }^{5}$ Nielsen H. Epileptic seizures following cervical myelography. Neuroradiology 1975;10:59-60.

${ }^{6}$ Lundervold A, Sortland O. EEG disturbances following myelography, cisternography and ventriculography with metrizamide. Acta Radiol [Diagn] (Stockh) 1977; Suppl 355:379-89.

${ }^{7}$ Gelmers J. Adverse side effects of metrizamide in myelography. Neuroradiology 1979;18:119-23.

${ }^{8}$ Teasdale E, Macpherson P. Incidence of side effects following direct puncture cervical myelography. Bed rest versus mobility. Neuroradiology 1983;25:85-6.

${ }^{9}$ Bassi P, Cecchini A, Dettori P, Signorini E. Myelography with iopamidol, a nonionic water-soluble contrast medium: Incidence of complications. Neuroradiology 1982;24:85-90.

${ }^{10}$ Kaada B. Transient EEG abnormalities following lumbar myelography with metrizamide. Acta Radiol [Diagn] (Stockh) 1973; Suppl 335:380-6.

1 Gonsette RE. Metrizamide as contrast medium for myelography and ventriculography. Preliminary and clinical experiences. Acta Radiol [Diagn] (Stockh) 1973; Suppl 335:346-58.

12 Gonsette RE. Biologic tolerance of the central nervous system to metrizamide. Acta Radiol [Diagn] (Stockh) 1973; Suppl 335:25-44.

${ }^{13}$ Skalpe IO, Amundsen P. Thoracic and cervical myelography with metrizamide. Clinical experiences with a water-soluble non-ionic medium. Radiology 1975; 116:101-6.

${ }^{14}$ Harrington MG, McGeorge AP, Ballantyne JPB. The effect of lumbar puncture on the electroencephalogram. J Neurol Neuros Psychiatry 1983;46:283-4. 